\title{
A propósito del puntaje de Rotterdam como predictor de pronóstico en el síndrome de Budd-Chiari
}

\section{About the Rotterdam Score as a predictor of Budd-Chiari Syndrome prognosis}

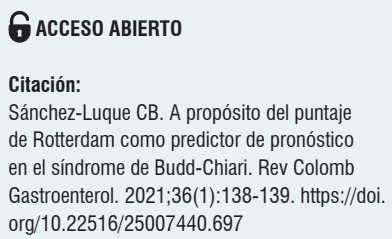

Palabras clave

Puntaje de Rotterdam, síndrome de Budd-Chiari.

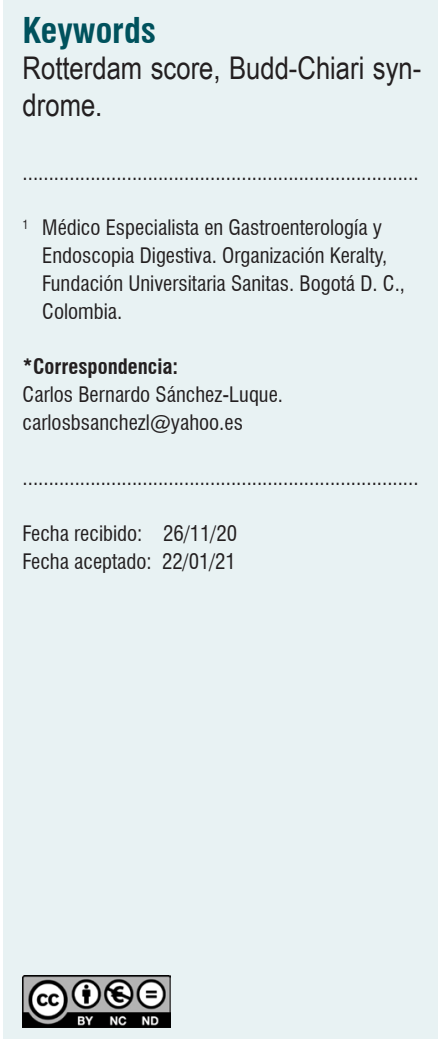

En 1845 el Dr. George Budd comentó por primera vez sobre 3 pacientes con obstrucción de las venas hepáticas en su seminario Enfermedades del hígado; en ese momento se pensó que la sepsis era la causa de la trombosis en dos pacientes. 53 años después, el Dr. Hans Chiari publicó una segunda serie de casos y, mientras ejercía como patólogo en Praga, observó a 3 pacientes con trombosis de las venas hepáticas; analizó estos casos junto con una revisión de la literatura que incluyó a otros 7 pacientes. A pesar de lo raro de la afección, enfocó la atención a "una enfermedad que podría conducir rápidamente a la muerte", y la llamó flebitis obliterante. Los 3 hígados estaban significativamente congestionados y necróticos, con congestión de la circulación portomesentérica y ascitis de gran volumen. La histología mostró una mínima reacción adventicia sin compromiso perivascular significativo. La trombosis se consideró una complicación de un proceso de endoflebitis que se producía en el contexto de la sífilis. En los años siguientes, esta hipótesis no se confirmó. Sin embargo, esta descripción es una piedra angular en el descubrimiento de lo que ahora se conoce como sindrome de Budd-Chiari (1).

He leído con mucho interés el estudio publicado recientemente por Muñoz-Maya y colaboradores en la Revista Colombiana de Gastroenterología, en el que se describen la etiología, manejo y resultados en una cohorte retrospectiva de 35 pacientes con diagnóstico de síndrome de Budd-Chiari. Debido a la rareza del síndrome de Budd-Chiari, no existen datos prospectivos en la literatura y los estudios más grandes hasta el momento se componen principalmente de datos recopilados de cohortes retrospectivas. Como lo mencionan los propios autores del estudio, es una de las series más grandes de pacientes con diagnóstico de síndrome de Budd-Chiari publicada en Colombia. En esta publicación, la gravedad de la patología se clasificó mediante la escala MELD (Model for Endstage Liver Disease) y por el puntaje de Rotterdam para predecir el éxito terapéutico y valorar la respuesta de forma más temprana, con el fin de no retardar el uso de medidas invasivas definitivas y mejorar el pronóstico (2).

A pesar de que el síndrome de Budd-Chiari es una entidad poco frecuente, tiene un pronóstico variable y puede tener una mortalidad temprana (que se define como la mortalidad a los 3 meses), hasta en el $20 \%$ de los casos: el rendimiento de los índices de gravedad como la clasificación de Child-Pugh, el MELD, la clasificación de Rotterdam y los criterios de Clichy para discriminar la mortalidad temprana aún es incierto. La puntuación de Rotterdam se determina con la siguiente ecuación:

$1,27 \times$ encefalopatía $+1,04 \times$ ascitis $+0,72 \times$ tiempo de protrombina índice internacional normalizado (INR) $+0,004 \times$ bilirrubina $\mu \mathrm{mol} / \mathrm{L}$ 
La ascitis y la encefalopatía hepática se puntuaron como presente [1] o ausente [0] y el tiempo de protrombina como mayor [1] o menor [0] que un INR de 2,3. La puntuación total osciló entre 0,02 y 4,03. De esta manera, se distinguen tres clases de pacientes:

- clase I (buen pronóstico): con puntuación total entre 0 y 1,1 ;

- clase II (pronóstico intermedio): con puntuación entre 1,1 y 1,5 ;

- clase III (mal pronóstico): con puntuación superior a 1,5 .

El mejor índice para discriminar la mortalidad a los 3 meses en pacientes con síndrome de Budd-Chiari fue la puntuación de Rotterdam, con un área bajo la curva (AUC) de 0,84 (intervalo de confianza [IC] del $95 \%: 0,68-0,98, p$ $=0,005)$. Por lo anterior, se ha concluido que la puntuación de Rotterdam es el mejor índice de discriminación para predecir la mortalidad a los 3 meses en el síndrome de Budd-Chiari y, por este motivo, debería utilizarse para determinar la urgencia del tratamiento (3).

Más recientemente, en cuanto al pronóstico de los pacientes con síndrome de Budd-Chiari, las guías de práctica clínica de la Asociación Europea para el Estudio del Hígado (EASL) mencionan que ha habido varios intentos para determinar parámetros o combinaciones de parámetros que pueden predecir el pronóstico en estos pacientes. Aunque todos estos índices de pronóstico son válidos para la evaluación de la supervivencia sin trasplante y la supervivencia sin terapia invasiva, su precisión predictiva es subóptima para su uso en pacientes individuales en la práctica clínica diaria. El desarrollo de carcinoma hepatocelular o la misma progresión de la enfermedad hematológica de base pueden modificar el pronóstico del síndrome de BuddChiari (4).

Se plantea un interrogante bastante trascendental: ies mejor usar anticoagulación, derivación portosistémica intrahepática transyugular (TIPS) o trasplante hepático para el tratamiento del síndrome de Budd-Chiari? Actualmente, no hay ninguna razón para elegir uno de estos en particular. Todos ellos son dignos de ser considerados en su manejo y los centros que tratan a estos pacientes necesitan tener una amplia experiencia en todas estas técnicas para asegurar el mejor tratamiento para los pacientes con síndrome de Budd-Chiari, por eso es que estos pacientes deberían ser derivados a los centros con mayor experiencia. Un manejo escalonado de terapias menos invasivas (anticoagulación) a terapias más invasivas (trasplante hepático), es el enfoque recomendado para los pacientes con síndrome de Budd-Chiari (5).

Se requiere de un diagnóstico oportuno para esta entidad por su alta tasa de mortalidad en ausencia de tratamiento. Las manifestaciones clínicas tempranas deben llevar a descartar otros diagnósticos, ya que en algunos momentos se puede lograr la resolución del síndrome al tratar las patologías concomitantes. Es importante tener en cuenta que el manejo depende de las condiciones de cada paciente y se sugiere el enfoque escalonado de lo menos a lo más invasivo, con el fin de brindar mejores desenlaces pronósticos en los pacientes, quienes, a pesar del tratamiento que reciben, deben llevar un seguimiento estricto (6). Se podría concluir razonablemente que los pacientes más enfermos requieren un trasplante de hígado urgente, pero no se puede excluir la posibilidad de que la derivación quirúrgica urgente o la colocación de TIPS también sean eficaces dependiendo de la experiencia local.

\section{REFERENCIAS}

1. Zanetto A, Pellone M, Senzolo M. Milestones in the discovery of Budd-Chiari syndrome. Liver Int. 2019;39(7):11801185. https://doi.org/10.1111/liv.14088

2. Muñoz-Maya O, Vergara Cadavid JA, Cajiao Castro L, Marín Zuluaga JI, Restrepo Gutiérrez JC, Santos Sánchez OM. Síndrome de Budd-Chiari: etiología, manejo y resultados en una cohorte de 35 pacientes del Hospital Pablo Tobón Uribe. Rev Colomb Gastroenterol. 2020; 35(3):280-6. https://doi.org/10.22516/25007440.431

3. Montano-Loza AJ, Tandon P, Kneteman N, Bailey R, Bain VG. Rotterdam score predicts early mortality in Budd-Chiari syndrome, and surgical shunting prolongs transplant-free survival. Aliment Pharmacol Ther. 2009;30(10):1060-9. https://doi.org/10.1111/j.13652036.2009.04134.x
4. European Association for the Study of the Liver. Electronic address: easloffice@easloffice.eu. EASL Clinical Practice Guidelines: Vascular diseases of the liver. J Hepatol. 2016;64(1):179-202. https://doi.org/10.1016/j. jhep.2015.07.040

5. Magaz M, Soy G, García-Pagán JC. Budd-Chiari Syndrome: Anticoagulation, TIPS, or Transplant. Curr Hepatology Rep. 2020;19:197-202. https://doi.org/10.1007/s11901020-00528-8

6. Arcila-Garcés LI, Correa-Vargas JD, Guerrero-Bermúdez C, Vélez-Marín M. Manejo de pacientes con síndrome de Budd-Chiari. Revisión de tema. Hepatología. 2020;1(1):55-67. 